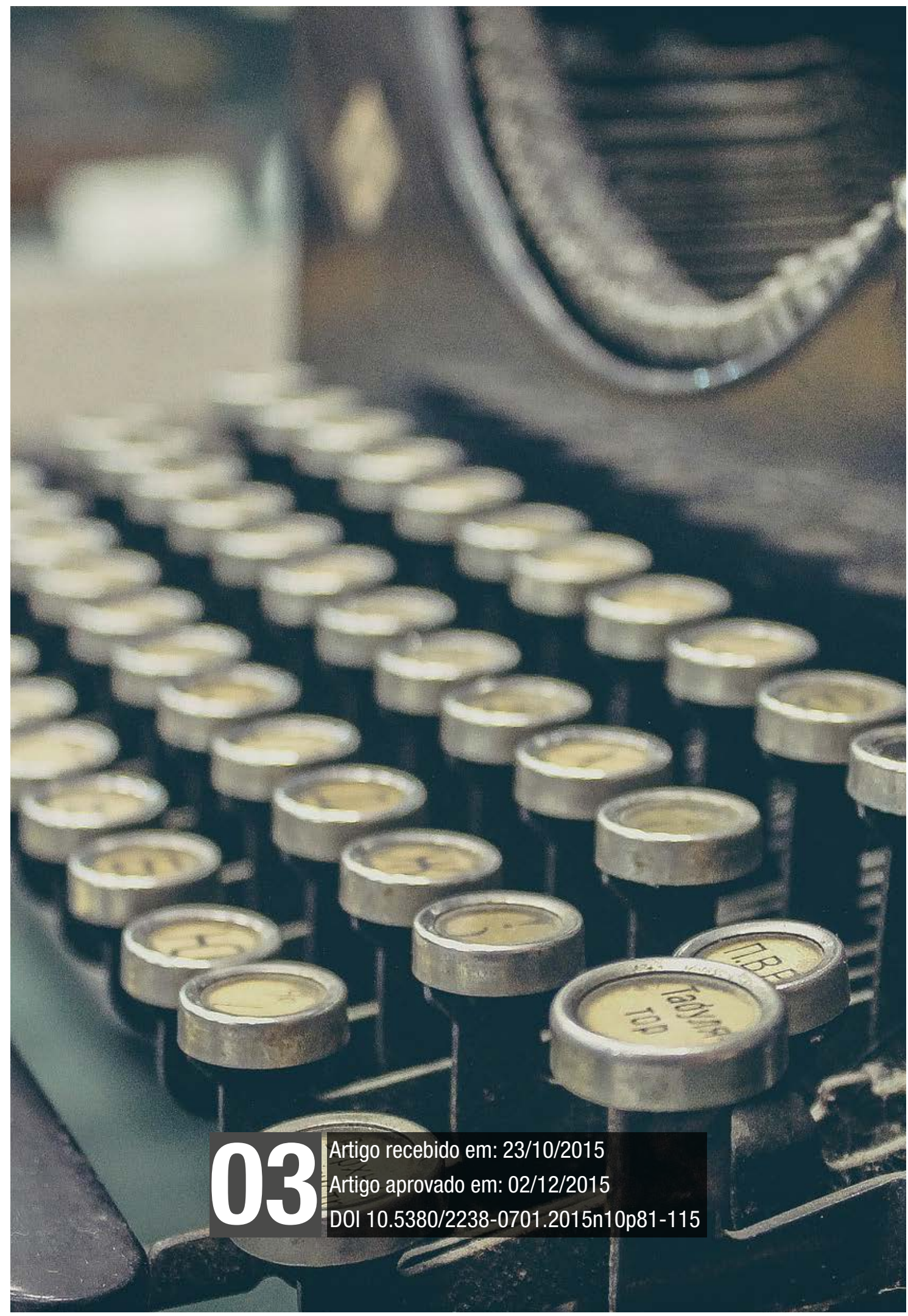


AÇÃO MIDIÁTICA, n. 10. Ju/Dez. 2015. Curitiba. PPGCOM-UFPR. ISSN 2238-0701 


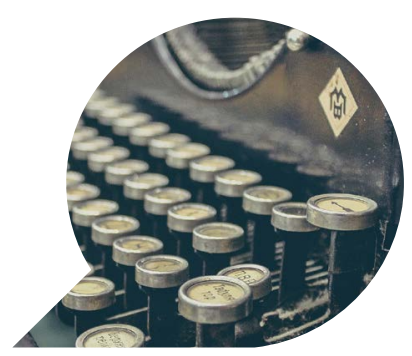

\title{
Al Huffington Post: une grille d'evaluation semiotique et situationnelle
}

\section{O Huffington Post: um quadro de avaliação semiótica e situacional}

\author{
Al Huffington Post: a semiotic and situational \\ evaluative grid
}

\section{JENNY MOUCHANTAF *}

\section{RAYAN HAYKAL}

Resumé: Le développement des pratiques de journalisme participatif redéfinit la frontière entre le journaliste professionnel et amateur dans lobjectif de démocratiser la production, lévaluation et la diffusion d'information. A travers une méthodologie sémiotique et situationnelle, une évaluation objective de la version Maghrébine du Huffington Post s'articule autour de trois axes: la crédibilité, le contenu et l’ergonomie de ce pure player. Une grille dévaluation détaillée confirme les critères de

\footnotetext{
* Professeure associée à l'université Antonine et collaboratrice scientifique au sein de l'Institut Langage et Communication de l'Université Catholique de Louvain, elle est spécialiste de l'analyse des discours médiatiques. mchantafjenny@gmail.com

* Dr. Rayan Haykal est Maître de Conférences et coordinateur du département des Sciences Economiques et des mémoires de MBA à la Faculté de Gestion et de Finance de l'Université La Sagesse. Il est le représentant de la Faculté aux Comités d'Ethique et de l'Assurance Qualité. Il détient un doctorat en sciences économiques de l'Université Paris 2 Panthéon-Assas: rayan. haykal@uls.edu.lb
} 
fiabilité, de solidité de contenu et de facilite d'accès à l'information, sans pour autant faire abstraction du manque de positionnement idéologique de cette expérience.

Mots-clés: Journalisme participatif; Evaluation sémiotique et situationnelle; Ergonomie.

Resumo: O desenvolvimento de práticas de jornalismo participativo redefine as fronteiras entre o jornalista profissional e o amador, no sentido de democratizar a produção, avaliar e disseminar a informação. Por meio de uma metodologia semiótica e situacional, foi realizada uma análise objetiva do Huffington Post Magreb, articulada em torno de três eixos: credibilidade, conteúdo e eficiência ergonômica deste pure player. Um quadro detalhado de resultados confirma os critérios de confiabilidade, de solidez de conteúdo e de fácil acesso à informação, sem ignorar, por outro lado, a falta de posicionamento ideológico desta experiência jornalística.

Palavras-chave: Jornalismo participativo; Análise semiótica e situacional; Ergonomia.

Abstract: The development of participatory journalism practices redefines the boundary between professional and amateur journalist aiming to democratising the production, evaluation, and dissemination of information. Through a semiotic and situational methodology, an objective assessment of the Maghreb version of the Huffington Post is built around three axes: credibility, content, and the ergonomic efficiency of this pure player. A detailed evaluation grid confirms the reliability criteria, the strength, and the ease of access to information without ignoring the lack of ideological positioning of this experience.

Keywords: Participatory Journalism; Semiotics and Situational Assessment; Ergonomics. 


\section{Introduction}

Larrivée des pures players depuis quelques années a bouleversé la donne médiatique et a donné naissance à ce qu'on appelle le journalisme alternatif. Ces protagonistes médiatiques sont uniquement présents sur le net, ils redéfinissent les conditions et les exigences d'un nouveau genre de presse. Le Huffington Post, pionnier dans le domaine a créé un concept qui occupe bel et bien une place médiatique solide sur la plateforme virtuelle. Ce type de sites d'information développe une approche de l'information et du journalisme qui soppose au schéma traditionnel des médias classiques, toujours appuyés sur un support papier. Aux prémices du passage vers le paper free, cette presse contemporaine compte prendre à contre-courant l'ambition annoncée par plusieurs mastodontes médiatiques visant ainsi, à convertir la façon d'informer, où l'instantanéité serait le maître mot.

Informations alternatives, sujets variés, actualités différentes et investigation aiguisée deviennent la base d'un média inspiré par une presse traditionnelle érigée par de grandes figures historiques, combinés à des journalistes novices qui ont vu le jour sur la toile. Bénévoles ou onéreux, ces nouveaux médias qui privilégient un rapport différent à l'information et à son lecteur gagnent petit à petit en reconnaissance mais ont du mal à être à égalité avec leur prédécesseur classique. Le journalisme numérique, malgré son accessibilité accrue et son déterminisme, endure encore d'un profond manque de légitimité auprès des caciques de la profession.

Le Huffington Post (HP) est un quotidien participatif né aux Etats-Unis. Diffusé uniquement sur le net, il a été créé par Arianna Huffington, Kenneth Lerer et Jonah Peretti en 2005. Il est alimenté par un grand nombre de journalistes rémunérés et plusieurs centaines d'internautes et de bloggeurs qui travaillent gratuitement. La production de l'information se fait sous forme de 3 déclinaisons : les articles publiés par des journalistes professionnels, les articles gratuits rédigés par des bloggeurs et des internautes et la reprise d'information d'agence de presse. Les contributions de bloggeurs regroupent de grands noms dans le monde politique et économique tels Bill Clinton, Michael Moore, Dominique Strauss-Kahn ou encore Bernard-Henri Lévy. Le HP mise sur les sujets à sensation ou l'information polémique qui lui assure un certain «buzz » sur la toile comme les images chocs, les scandales, les sujets à caractère sexuel... 
Le Huffington Post existe sous plusieurs éditions internationales qui se sont graduellement intégrées dans la sphère du journalisme participatif. Al HP Maghreb a vu le jour en Juin 2013.Cette édition en français diffuse l'information de la Tunisie, le Maroc et l'Algérie. Le lancement de lédition africaine est survenu au moment où la région du Maghreb a connu d'importantes transformations sociales, économique et politique. Selon la présidente et fondatrice Arianna Huffington, lédition du Maghreb est plus qu'une plateforme journalistique internationale, c'est un journal d'interactions offrant de nouvelles possibilités pour la nouvelle génération de citoyens blogueurs générés et accentués par la mutation de la région. Lédition africaine est la seconde édition asiatique du journal, après la création de l'édition japonaise en juillet 2013.

La journaliste Alix Etournaud et l'entrepreneur Farès Mabrouk sont les partenaires fondateurs du "Al Huffington Post Maghreb". Les trois rédactions d'Al HP sont composées de 8 journalistes chacune; Kader Abderrahim assure la direction éditoriale du site qui est un mélange d'informations en temps réel, de blogs, de contributions, de vidéos et de présence sur les réseaux sociaux. Le HuffPo s'est hissé, pour des dizaines de millions de gens, au cœur de leur accès à l'information. Le HP est critiqué notamment aux États-Unis pour son style trop sensationnaliste et pour l'utilisation de clickbaits, consistant à s'appuyer sur des éléments sensationnels au détriment de la qualité ou de l'exactitude dans le but de générer des revenus publicitaires.

Cet article propose une approche sémiotique et situationnelle pour réaliser une évaluation objective du contenu d'Al HP. Après une revue de littérature sur le journalisme participatif, nous utiliserons une grille d'évaluation s'articulant sur 3 axes (Crédibilité, Contenu et Ergonomie) pour analyser la crédibilité de la production de l'information, la solidité du contenu et la facilite d'accès à l'information.

\section{Revue de Litterature}

1. Un mouvement aux frontières mal définies

Le journalisme participatif donne au lecteur un rôle actif dans le processus de collecte, de reportage et de dissémination de l'information d'actualité (Bowman \& Willis, 2003). Le récepteur devient 
émetteur et même un média. Il produit l'information, la juge, l'évalue et la diffuse en subissant des coûts négligeables. Le papier d'édition est remplacé par la page web, la caméra par le smartphone et le journaliste traditionnel par un quidam. La notion de journalisme participatif et citoyen vient supplanter la dimension participative par un ancrage démocratique en dotant le citoyen-journaliste d'une responsabilité civique dans la production d'information. Les internautes utilisent des moyens d'édition personnelle (blogs) ou collaborative (wikis) dans le but d'atteindre un idéal de justice dans l'accès et la production d'information. La participation s'effectue à 3 niveaux: la conception (production écrite), l'édition (la vérification de la fiabilité) et la distribution (REBILLARD, 2011). Lémergence du journalisme participatif s'inscrit dans un débat intellectuel (le fameux débat Lippman-Dewey) entre deux visions du journalisme. La première (celle de Lippman) considère le journalisme comme un système hiérarchisé, vertical entre producteurs professionnels d'une part et consommateurs d'information d'autre part. Selon cette vision, les medias interprètent l'information pour le compte des consommateurs étant donné que ces derniers ne sont pas en mesure de le faire eux-mêmes. La seconde (Dewey) considère le journalisme comme un système collaboratif qui se développe en démocratie et dans lequel le citoyen est invité non seulement à réfléchir et produire mais également à évaluer et seconder les professionnels du journalisme dans la chaine de production de l'information (HERMIDA et al., 2011). In extremis, le journalisme s'assimilerait à une " conversation citoyenne »(ALTERMAN, 2008) qui supposerait une éthique implicite de la part des journalistes-citoyens allant de la simple observation, au filtrage, à l'édition, la distribution et l'interprétation du contenu. La première expérience notable dans l'explosion des medias participatif revient à l'expérience du site coréen OhMyNews (KIM \& HAMILTON, 2006), puis les plateformes se sont multipliées, internationalisées, régionalisées, traduites et copiées ${ }^{1}$. Les différents sites affichent leur orientation prioritaire qui consiste à engager le citoyen dans le processus en le mettant au centre de la dynamique de production. Des slogans tels que: "Every citizen is a reporter", "Libération, c'est vous", "We, the média” donnent le ton de ces médias participatifs. La grande majori-

1 Enquête ouverte, Enlarge your Paris, Voix-Zines, StoryMaker, Bayosphere, Desourcesure, Vudesquartiers, SportVox, Mediapart, Centpapiers.... 
té de ces médias fonctionne en fait selon le principe de mixité "pro/ am" entre journalistes-professionnels et de journalistes-amateurs. Plusieurs critères contribuent à l'identification d'un " pro » comme la carte professionnelle, l'expérience, la notoriété, l'expertise; le critère le plus utilisé reste la salarisation de la contribution journalistique. La pseudo-identité du journaliste-amateur se mélange avec le militantisme politique et social, l'utilisation des réseaux sociaux à des fins commerciales ou comme vitrine d'exposition. Une combinaison entre l'intelligence individuelle et collective brouille les frontières entre audience et auteurs (COVER, 2006) et ré-agence la circularité des flux d'informations.

L'objectif principal des médias participatifs, qu'il soit déclaré ou non, est de casser le réseau de liens directs ou indirects entre les médias, les groupes industriels et les hommes politiques. Cette césure permettrait de casser l'hégémonie des médias traditionnels qui sont de moins en moins indépendants financièrement et remet le contenu de l'information comme critère principal du choix des médias. En effet, les médias traditionnels dont l'indépendance intellectuelle est contrainte par le financement publicitaire offrent un discours centriste (ou du moins qui ne heurte pas les lecteurs) afin de gagner en part de marché. Si cette tendance n'est pas encore visible, elle deviendrait de plus en plus prononcée surtout avec l'accroissement de la concurrence sur un même marché. La gratuité affranchit justement les médias de cette obligation de profitabilité et permet de remettre le processus démocratique du côté des citoyens-journalistes (PELISSIER et CHAUDY, 2009).

Par ailleurs, le journalisme participatif contribue à léclatement des formes traditionnelles de capital (DE ROSNAY, 2006) en mettant le capital informationnel comme richesse créée et cumulée par les journalistes-citoyens. Ce capital n'appartient pas à une classe donnée, mais à tout le monde. Il peut servir d'un tremplin extraordinaire au partage et à la transmission de richesses. La propriété collective, non excluable et non rivale de ce capital redéfinit le rapport économique des classes entre ceux qui possèdent l'information et ceux qui en sont privés. Dans les sociétés industrielles, la détention du capital physique et financier était la clé du développement, mais générait des inégalités de classe. Dans les sociétés modernes, la détention de l'information est la clé des investissements et donc du développement. Cette détention est ouverte à tout le monde. 
Cependant, la structure du marché des médias participatifs reste suffisamment compartimentée pour permettre une recherche de rentes. A la concurrence au niveau du contenu sajoute une segmentation par le marquage politique et une différenciation selon le public visé, la priorité donnée au contenu de l'information, la présentation du site, le slogan adopté par la rédaction, la spécialisation des rubriques, la volonté d'attirer la clientèle (surtout pour les sites payants), le choix des titres, la scénarisation, la dramatisation des articles. Il existe énormément de niches de marchés sur lesquelles les médias peuvent fidéliser leur clientèle et assurer leur durabilité. La diversité du contenu permet aux consommateurs d'information de mieux choisir, comme elle peut aussi bien générer une compétition entre des informations faibles en contenu (CHAMPLIN et KNOEDLER, 2006).

Ce marché produit ses propres mécanismes d'autorégulation qui font abstraction de l'intervention de l'Etat. L'horizontalité des réseaux d'échange d'information évite la verticalité du pouvoir de censure et de régulation. Quel rôle devrait donc jouer l'Etat dans ce contexte? Il peut jouer le rôle d'un garde-fou dans la limitation des défaillances marchandes de cet « écosystème » informationnel.

\section{Des limites qui fragilisent lécosystème:}

La question fondamentale qui met en danger le développement des médias participatifs est justement l'élargissement de la participation. Qui produit l'information? Le mythe de gens ordinaires, témoins d'évènements et de faits d'actualité qui se mobilisent pour produire une information objective reste loin d’être réalisé. Selon des études empiriques menées par des sites comme OhmyNews et Agoravox, le profil des citoyens-journalistes n'est pas aléatoire. Pour une grande partie de ces rédacteurs, le profil est le même: éduqués, citadins, jeunes, exerçant des professions intellectuelles, ayant des habitudes de rédaction et de publications (pas nécessairement d'articles de presse) et même des journalistes de formation. Lidéal de justice est loin dêtre vérifié par ce biais de sélection. En plus, ce marquage social se dote d'un marquage militant. Les rédacteurs qui potentiellement auraient pu être cadrés dans un média traditionnel se trouvent relégués à une plateforme pseudo-participative. Le slogan de «tous journalistes » reste donc l'apanage d'une minorité sociale. Ces ingénieurs du social (BELL, 2003) sont en effet un petit groupe qui utilise 
les médias participatifs comme exutoire. Pour assurer la pérennité des medias participatifs, il ne s'agit pas d'ouvrir la porte à la diversité d’opinions mais à une véritable collaboration dans la production d'information, loin de l'excessive vulgarisation scientifique.

En plus, une bonne partie de la population se trouve privée des moyens techniques, administratifs ou linguistiques pour prendre part à ce militantisme journalistique. Au-delà du fait que dans les pays sous-développés, l'infrastructure technologique reste rudimentaire, il n'est pas à exclure que même dans les sociétés dotées de moyens techniques avancés, la sociabilité numérique ne soit pas à la portée de tout le monde.

Un autre problème émerge concernant la fiabilité de l'information. Le fameux «check, double-check » de la profession journalistique qui consiste à vérifier et croiser les sources d'information se trouve marginalisé par l'amateurisme des rédacteurs-internautes. A cet effet, certains sites comme Rue89 ont mis en place une architecture d'accompagnement qui consiste à filtrer les articles et à retenir ceux qui sembleraient les plus pertinents. Encore une fois, l’idéal de démocratisation de la production d'information semble limité par les mécanismes d'autorégulation du journalisme participatif. Une injonction économique ou politique au sommet de la chaîne de production reconfigure la singularité propre à la procédure.

Au niveau de la diffusion de l'information, les internautes (journalistes amateur ou non) sont capables via les réseaux sociaux à séchanger des informations allant d'une simple conversation par email au partage d'articles ou d'hyperliens intégrés à leurs messages. Ils contribuent donc à la transmission d'une information de seconde main dont la source n'a pas été vérifiée. Et même si la source est vérifiée, rien ne garantit la véracité de l'info. Du temps des médias traditionnels, la publication d'une rumeur ou d'une fausse information étant corrigée par un simple encart d'excuse dans lédition du jour suivant. Cette limite ne devrait pas cependant pousser l'Etat à légiférer un statut juridique spécifique au journaliste-amateur d'autant plus que les contributeurs professionnels eux-mêmes ne sont pas tous couverts par une carte de presse. La multiplication de la diffusion de rumeurs ou d'infos non-fondées fragilise le système en entier.

Enfin, le principe de mixité se heurte également à la viabilité des modèles économiques des différents médias entre la gratuité, le recours aux recettes publicitaires, au mécénat ou au sponsoring. 
Le peuple-source "crowd-sourcing " au niveau de la production devrait-il également sétendre au financement afin d'assurer une pérennité de ces médias. Encore une fois, le fait de demander aux lecteurs de financer ne remet-il pas les médias participatifs dans la même logique industrielle des médias traditionnels sous le diktat des groupes publicitaires ? De plus, un ajustement par le bas peut toucher la contribution des journalistes eux-mêmes. Le débat porterait encore une fois une dimension déontologique que le concept même de média participatif est supposé en affranchir les producteurs d'information. Si l'Etat est capable de réguler le marché du côté de l'offre (en refusant d'accorder des licences, en majorant les prix ou les abonnements), son action devrait sétendre aussi du côté de la demande en faisant respecter le droit du lecteur à une information non biaisée (BAKER, 1998).

\section{Methodologie de Travail}

MUCCHIELLI (2008) a travaillé sur la sémiotique situationnelle, méthodologie qui analyse le site web en partant de la situation de communication. Les propositions de communication du site web sont analysés afin de trouver celles qui s'avèrent comme les plus expressives en situation d'usage pour répondre à notre objectif de questionner et d'évaluer le contenu du HP - Maghreb. Dans le cadre de cet article, nous définissons le principe fondamental de la sémiotique situationnelle. Un deuxième point est dédié à l'application de la méthode pour l'évaluation selon les trois critères d'évaluation et d'analyse: identification des ressources, les auteurs et les types de documents diffusés. Enfin, nous concluons sur l'intérêt de notre proposition méthodologique dans la perspective d'une meilleure opérationnalité des méthodes qualitatives.

La sémiotique situationnelle, issue de la sémiologie contextuelle (MUCCHIELLI, 1998, 2005), est une méthode des sciences humaines qui trouve ses origines au sein de la sociologie compréhensive vu qu'elle vise à la compréhension des phénomènes sociaux complexes (MORIN, 1990). Développée par Wilhelm Dithley à partir des années 1880, l'approche compréhensive distingue deux postures épistémologiques : le discernement et l'interprétation. À la suite d'Husserl et de Weber, c'est Alfred Schutz, au milieu des années 1950, qui a développé l’approche compréhensive ou « étude du fait 
humain et social ». Il postule que pour comprendre les « choses» de leur monde, les acteurs sociaux recourent inévitablement à une mise en situation (Ibid, pp. 55-56). Laccent est mis sur la situation qui apparaît comme subjective pour tel ou tel acteur social puisqu'entièrement dépendante de son système de pertinence. Cette activité de « compréhension », que l'École de Palo Alto appelle la réalité de second ordre, est à son tour mise en œuvre par le chercheur pour comprendre comment l'acteur social interprète le monde dans son vécu situationnel. Nommée empathie, ou attitude compréhensive, cette méthode nous permet en tant qu'être humain de "s'immerger dans le monde subjectif d'autrui » (Ibid, p.55) sans pour autant le vivre dans notre propre réalité. La sémiotique situationnelle ne s'intéresse pas à lémission ou au contenu des messages, mais au phénomène d'émergence de sens en lien avec une "contextualisation naturelle faite par l'acteur ». Le sens d'une communication n'est donc pas, ici, un résultat déterminé par une relation de cause à effet mais considéré comme une émergence par et dans une situation elle-même définie par un ou plusieurs acteurs sociaux (HEID et MELIANI, 2009).

\section{Analyse des Resultats}

L'objectif de notre recherche est de différencier l'avantage que le HP- Maghreb offre par rapport aux autres sources d'informations disponibles sur le net et de juger de la crédibilité des outils de recherches et de leur résultat. En vue d'évaluer le contenu du site participatif HP, nous avons décelé trois grands critères dévaluation et d'analyse. La crédibilité, le contenu, et l'ergonomie. Ainsi, nous pourrons mieux évaluer les ressources, les auteurs et les types de documents trouvés.

\section{Axe 1: La crédibilité}

\section{A. Responsabilité intellectuelle}

1. Mention des noms, logo et références

Le titre, logo, date et pays d'édition apparaissent en début de page pour les trois versions du Huffington Maghreb. 


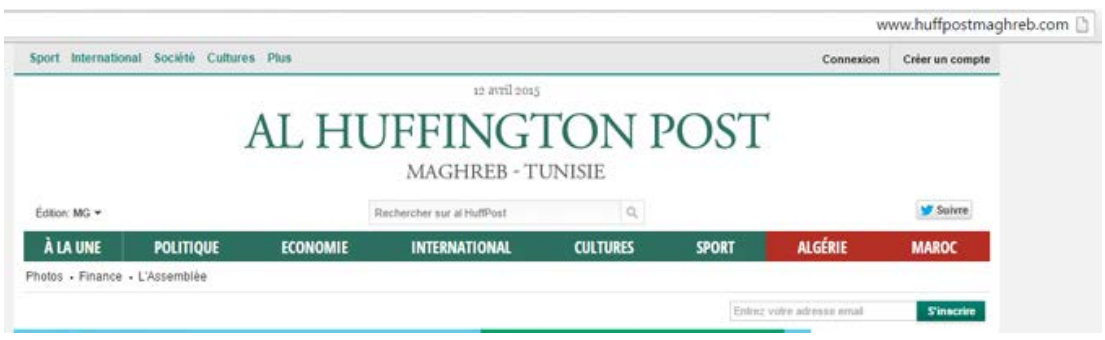

Figure 1: Page d'accueil du Al Huffington Post Magreb-Tunisie

2. Mention des noms et responsabilité de l'auteur pour chaque document du site

Dans cette catégorie, nous avons sélectionné trois différents articles des 3 versions du HP appartenant à la rubrique " blogs". Les auteurs rédigent différents articles, blogs et opinions, leurs noms et leurs activités professionnelles apparaissent en début d'article.

Article n.1: La dépression n'est pas un facteur de risque de criminalité et les antidépresseurs sauvent plus qu'ils ne tuent-21 avril 2015- le blog Rubrique: santé

Auteurs: Jean-François Costemale- Médecin psychiatre, Chef de clinique Gilles Martinez- Médecin psychiatre

Marion Plaze- Psychiatre

Article n.2: 20 ans de l'OMC à Marrakech: Comment faire profiter les peuples africains de la libération des échanges mondiaux- 10 avril 2015- Huff Maroc Rubrique: économie

Auteur: Hamid Bouchikhi

Article n.3: Espoirs d'alternance- Blog- 12 avril 2015-

Rubrique: littérature

Auteur: Amokrane Nourdine 
3. Statut de l'auteur: est- il reconnu dans son domaine ou sa discipline?

Les 3 auteurs évoqués dans l'exemple précédent sont reconnus dans leur secteur d'activité. Dr Jean-François Costemale-Lacoste est un médecin psychiatre. Consultant universitaire du Service Hospitalo-Universitaire du Pr Krebs au sein du Centre Hospitalier Sainte-Anne à Paris.

Gilles MARTINEZ est lui aussi médecin psychiatre. Actuellement Chef de Clinique dans le Service Hospitalo-Universitaire du Pr KREBS au Centre Hospitalier Sainte Anne. Ce dernier est responsable d'une unité d'hospitalisation spécialisée dans la prise en charge des troubles schizophréniques et de l'humeur résistants. Marion PLAZE est responsable de la consultation universitaire dédiée aux patients souffrant de pathologies psychiatriques résistantes. Elle est également à la tête d'une cellule de recherche en imagerie cérébrale au sein du Centre de Psychiatrie et Neurosciences (Inserm, Paris Descartes).Hamid BOUCHIKHI est professeur de management et d'entrepreneuriat. C'est un chercheur reconnu au niveau européen dans le domaine de la théorie des organisations, l'entrepreneuriat et l'innovation managériale. Il est également le fondateur et le directeur académique de l'Essec Entrepreneurship Center. Après un passage à l'École Polytechnique Fédérale de Lausanne en Suisse, Amokrane NOURDINE enseigna un peu les maths dans des lycées algériens. Il est auteur de Il y a 50 ans... l'Algérie et Soliloques et passions d'exil.

\section{B. Contexte de création}

\section{Source de financement, indépendance de l'auteur}

La publicité est la première source de financement du site Huffington Post Maghreb. Chaque pays a une cible marketing précise et en dépit de cela, le Huffington publie les différentes bandes annonces de marques spécifiques.

\section{Nature du site}

Le Huffington Post Maghreb se présente au public comme un site commercial. Nous constatons cela dans la maquette du site et sous la vignette « contacter nous ». Léquipe éditorial du site permet 
aux commerçants de déposer leurs annonces publicitaires, commerciales, et ouvre même la porte aux partenariats et aux parrainages.

\section{Statut de l'information}

Le Huffington Post est un site gratuit, l'information est gratuite et non payante. Les internautes ont un accès illimité à l'intégralité des articles et des blogs, contrairement à site d'information classique comme le Courrier International qui publie l'introduction des articles et invite les gens à s'abonner à un forfait mensuel pour avoir accès à toute la publication. De plus l'abonnement à la Newsletter du Huffington Post est gratuite.

\section{Présence de publicités en lien ou non avec le site}

Le financement du Huffington Post se fait à travers la publicité. Des annonces d'offres de banque, d'assurances, de forfaits téléphoniques sont placées à la Une distinctement, en haut de page ou dans les colonnes à droite de la page principale.

\section{Existence d'un comité éditorial}

Pour en savoir plus sur l'équipe éditoriale, il suffit de cliquer sur la vignette "A propos de nous", nous y trouvons les noms des différentes personnes qui contribuent au bon fonctionnement du site. Léquipe est divisée comme suit : le directeur éditorial, le rédacteur en chef, deux journalistes généralistes, journaliste et responsable du blog et un responsable de la gestion de l'activité, des réseaux sociaux, des lecteurs et des différentes interactions avec le public. Ce dernier peut également gérer le nombre de lecteurs et de visiteurs.

\section{Fraicheur}

\section{Actualisation régulière des documents du site}

Le site du Huffington, se présente comme un site quotidien, avec des articles publiés et actualisés le jour même. Le site publie l'actualité dans les différentes rubriques, santé, people, littérature, économie et même des sujets tendances sur les réseaux sociaux. 
2. Mention de la date de création, date de dernière mise jour et éventuellement date dernière révision

Au-dessus de chaque article figure la date de publication et la date et l'heure de mise à jour de cet article.

Exemple:

\section{Article:}

«De l'autoritarisme en Tunisie (1/2): Rien de nouveau sous le soleil! » Auteur : Farhat Othman : Juriste, politiste, chercheur en sociologie et ancien diplomate Publication: 24/04/2015 14h44 CEST Mis à jour: il y a 20 minutes Lien de larticle: http://www.huffpostmaghreb.com/farhat-othman/ de-lautoritarisme-en-tuni_b_7116528.html?utm_hp_ref=maghreb "La sagesse antique reprise dans l'Ecclésiaste de la Vulgate (chapitre 1, verset 9), affirme avec raison: Nihilnovi sub sole, ainsi que le traduit le titre. C'est aussi le cas en Tunisie et il est donc temps de tenir un langage de vérité. «

\section{Audience du Site}

\section{Possibilité de connaître le nombre de visiteurs}

Le site ne mentionne pas le nombre de visiteurs, mais il est possible d'utiliser une approximation à travers le nombre de followers sur Twitter ou de likes sur Facebook.

2. Possibilité de connaître le nombre de documents ou de pages visualisées

Nous pouvons estimer approximativement le nombre de personnes qui consultent les articles publiés en remarquant les chiffres de partage de l'article sur les réseaux sociaux: 


\section{Le SNJT propose un conseil de la presse écrite et électronique}

HuffPost Tunisie avec TAP

Publication: 03/05/2015 12h41 CEST | Mis à jour: il y a 57 minutes

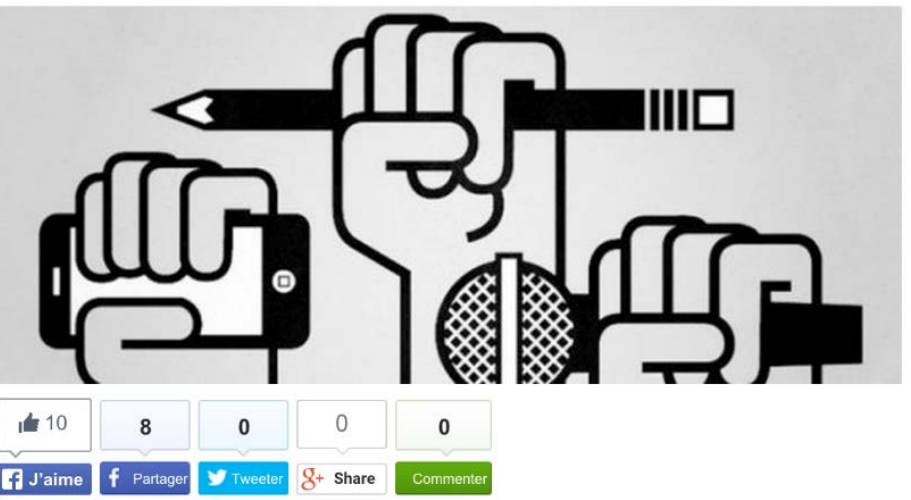

MÉDIAS - Dans son rapport annuel présenté à la veille de la célébration de la journée internationale de la liberté de la presse, le syndicat des journaliste (SNJT) a appelé samedi à la création d'un conseil de la presse écrite et électronique.

Le syndicat a déploré la multiplication des atteintes à la déontologie journalistique, à travers plusieurs phénomènes:

Figure 2: Exemple de traçabilité d’un article

Exemple de traçabilité d'un article:

- 10 personnes ont aimé l'article

- 8 personnes ont partagé l'article sur leur compte Facebook,

- 0 personne a tweeté

- 0 personne a partagé sur Google plus,

- 0 personne a commenté l’article sur la page du site.

Notons qu'une bonne partie des lecteurs liront l'article sans laisser une trace visible, la détection de ses lecteurs ne peut être dévoilée que par l'administrateur du site. Mais comme les chiffres présents sur les réseaux sociaux apparaissent au public, nous pouvons estimer le nombre de visiteurs du site d'environ 55000 personnes dues aux nombres de Likers de la page Facebook.

Le HP Maghreb possède une page sur Twitter pour chaque pays présentant différents chiffres en termes de « Followers » 
- Tunisie: 1399 Followers

- Algérie: 2093 Followers

- Maroc: 2360 Followers

Adoptant la même méthode que celle de Facebook, nous pouvons évaluer le nombre de followers pour chaque site d'après le nombre de visiteurs, due au manque d'accès au back end du site, réservé au gérant du site.

\section{E. Accessibilité du site}

1. Référencement du site sur internet et dans les medias spécialisés ou généralistes

Le Huffington Post Maghreb, a vu le jour le 18 aout 2013 en Tunisie, et s'est intégré très rapidement dans les pays du Maghreb Arabe. Depuis sa naissance dans cette région du monde, beaucoup de médias participatifs, spécialistes et généralistes ont pris ce site comme référence. Des blog participatifs comme "The fly on the wall », de publications spécialistes comme «Bloomberg Business » ou de presse généraliste comme Le monde et France 24, ces derniers ont tous annoncé le lancement du site participatif au Maghreb « Le Huffington Post»

\section{Exemple:}

Site: www.thewrap.com

Titre: Huffington Post Expands to Africa

Auteur: Sarra Morrison

"The Huffington Post has launched another international vertical - $\underline{\mathrm{Al}}$ Huffington Post Maghreb, covering Algeria, Morocco and Tunisia. Arianna Huffington, the site's editor-in-chief and president of AOL's Huffington Post Media Group, made the announcement on her HuffPo blog - a medium that will be heavily featured in HuffPo Maghreb’s first days."

Après l'annonce de la naissance de ce site, Le Huffington Maghreb est devenu une référence dans cette région du monde. 
France 24

Titre de l'article: Tunisia’s Amina quits 'Islamophobic' Femen

Lien:http://www.france24.com/en/20130820-jailed-tunisia-activist-amina-leaves-islamophobic-femen-organisation/

Reference: "I do not want my name to be associated with an Islamophobic organisation," she told the Maghreb edition of the Huffington Post.

2. Nombre de liens pointant vers le site:

Le software " siteexplorer.info » est un outil en ligne, qui permet de savoir le nombre exact de liens associés à un site. Les chiffres révélés par cet outil pour le HP Maghreb sont de 79 liens pointant vers le site, venant de 17 pays différents en particulier des Etats- Unis, de la France et du Canada. 29 mots clés sont utilisés dans les outils de recherche tel que : Maghreb/ Huffpost/infos dernières minutes.

\section{Référencement réussi dans les principaux moteurs de recherche}

Le référencement d'un site consiste à le faire connaître auprès des internautes, pour qu'il devienne repérable et visible sur les moteurs de recherche. En utilisant des mots clés comme "Info Maghreb », le lien du site Huffington Post apparaît en cinquième place sur la première page de Google, ce qui signifie, qu'il est toujours parmi les 10 premiers liens d'information portant sur le Maghreb. Sur Yahoo et avec les mêmes mots clés "Info et Maghreb" le lien pointant vers le Huffington Post Maghreb napparait pas dans la liste proposée par le moteur de recherche. Ceci dit, il se peut que l'équipe qui gère la visibilité en ligne du Huffington ne cible pas tous les moteurs de recherches.

\section{IV.2. Axe 2 : Contenu}

\section{A. Exactitude et exhaustivité}

1. Evaluer le niveau d'exhaustivité, de précision et d'exactitude des informations. 
Dans son document « Evaluation de l'information présente sur Internet " L'INSA (Institut National des Sciences Appliquées Lyon), propose une grille d'évaluation d'exactitude et d'exhaustivités des informations publiées en ligne, surtout les informations gratuite, comme le HP Maghreb, où le producteur publie les informations sous sa propre responsabilité, et comme nous l'avons observé précédemment avec l'absence d'un comité scientifique pour la vérification du contenu. Les sources utilisées pour rédiger les documents appartiennent-elles aux normes internationales ? Dans certaines de ses rubriques, le Huffington Post, prend comme référence l'Agence France Presse comme source d'information, LAFP qui se présente comme source fiable d'information et d'actualité médiatiques, et dans le sens inverse, France 24 une autre chaîne médiatique à réputation fiable, se réfère au Huffington Post dans une de ces publications.

\section{Exemple 1:}

Article: Source AFP, Publié sur Huffington:

Titre: « Tunisie: Deux soldats et dix “jihadistes" sont morts lors d'affrontements, près de la frontière algérienne " Auteur : HuffPost Tunisie/AFP

Lien: http://www.huffpostmaghreb.com/2015/04/24/tunisie-soldats-affrontements-jihadites_n_7136028.html

"SÉCURITÉ- De récents affrontements entre l'armée tunisienne et des "jihadistes", ont fait de nouvelles victimes dans les rangs de l’armée.

En effet, deux soldats tunisiens et dix jihadistes ont été tués lors d’affrontements dans un massif montagneux près de la frontière algérienne où une opération militaire est en cours depuis mercredi, a indiqué vendredi à l'AFP le ministère de la Défense. »

Exemple 2: 
Article: Source Huffington Post Maghreb, publiésur France 24:

Titre: « Tunisia’s Amina quits 'Islamophobic' Femen »

Auteur: AFP

Lien:

http://www.france24.com/en/20130820-jailed-tunisia-activist-amina-leaves-islamophobic-femen-organisation/

" "I do not want my name to be associated with an Islamophobic organisation," she told the Maghreb edition of the Huffington Post."

Le site propose-t-il un corpus cohérent et complet, ou bien est-il encore «en construction?

Le HP présente une maquette électronique complète et exhaustive, mais plusieurs liens se trouvant sur le site, nous dirigent vers les sites homologues de la Tunisie, et vice versa.

Par défaut l'adresse du site http://www.huffpostmaghreb.com/ nous oriente à la page principale Maghreb-Tunisie : Les rubriques en rouge « Algérie et Maroc » du menu de navigation nous renvoie respectivement à: http://www.huffpostmaghreb.com/algerie/ et http:// www.huffpostmaghreb.com/maroc/

Il y a 3 équipes rédactionnelles (Tunisie, Maroc et Algérie). Chaque équipe a un directeur de la rédaction, or la directrice de la rédaction de la Tunisie est la coordinatrice éditoriale des 3 équipes $^{2}$. Aucune page, rubrique ou section du site est " en construction ». Le site est complètement exploitable, avec neuf rubriques : la Une, culture, sport, international, et économie. En tête de la page d'accueil, le site propose des applications pour Smartphone, en version IOS ou ANDROID et même pour Windows. Le site est muni d'un moteur de recherche interne. A ne pas oublier également les flux $\mathrm{RSS}^{3}$ du site: http://www.huffpostmaghreb.com/syndication/

2 http://www.huffpostmaghreb.com/p/huffpostmaghreb-a-propos-de-nous.html.

3 Le RSS permet de s'inscrire à un site web au lieu de le visiter. Ce mode d'accès gagne en popularité auprès des internautes, en particulier des adeptes de technologies, des blogueurs invités et des nouveaux accros à Internet qui s'intéressent aux blogs et aux actualités. 


\section{SUIVRE AL HUFFPOST}

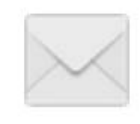

f
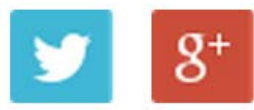

Votre e-mail

$\checkmark$ Newsletters

Recevez les meilleurs articles et contributions par mail chaque jour.

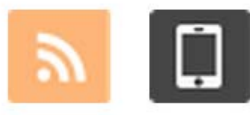

Je m'inscris !

Figure 3 : Connexion aux réseaux externes

2. Evaluer le degré d'originalité des informations proposées: sont-elles trouvables ailleurs, ou sont-elles exclusives

En se référant à l'INSA et sa grille dévaluation du contenu des sites gratuits, on peut se poser la question suivante: «Que rapporte de plus ce site par rapport aux autres sources d'informations disponibles déjà sur le net ou ailleurs? » Pour une étude plus efficace, nous allons nous inspirer de la rubrique économique, dans un cadre temporel similaire. Nous pouvons remarquer, que les articles publiés sur Rue 89 sont diversifiés et s'attaquent à des sujets internationaux, abordant les sujets du tourisme " hôtels, Booking.com, festivals de musique... " Alors que le Huffington Post est plus concerné par les sujets Maghrébins, publiant dans la rubrique économiques trois articles évoquant l'économie locale en l'occurrence en Tunisie et au Maroc.

Exemple de sujet traité au Huffington:

Et si le secteur formel marocain était le véritable obstacle au développement économique? Le moment est venu pour soutenir la Tunisie et de mettre laccent sur léconomie ${ }^{4}$

4 http://www.huffpostmaghreb.com/news/economie 
Exemple de Rue 89:

Avec la caisse automatique, la caissière devient un peu flic.

La richesse mondiale a doublé depuis $2000^{5}$

Donc le Huffington Post, dans sa version Maghrébine, se présente comme une source participative, originale d'informations quotidienne ayant rapport avec cette région du monde. Avec bien sûr, des articles et informations diversifiés et généralistes dans toutes ses rubriques.

Prenons un autre exemple: Le Huffington Post publie des sujets en exclusivité, dirigé par l'institut OpinionWay pour le site Monster.fr,

«Dans un sondage mené par l'institut OpinionWay pour le site Monster. fr que Le HuffPost publie en exclusivité ce jeudi 9 avril 2015, 1001 salariés français ont dû choisir entre quatre personnages de la série Game of Thrones, celui ou celle avec lequel ils préféreraient travailler parmi Daenerys Targaryen, Tyrion Lannister, Jon Snow et Jaime Lannister ${ }^{6}$

\section{B. Qualité de la langue}

2.1. Qualité de l'orthographe et de la grammaire/ Qualité des traductions éventuelles : mention de la source originale, du traducteur

En ce qui concerne la traduction des publications, et mention de la source originale, le Huffington Post Maghreb publie initialement en Français, mais la plupart du temps il choisit de se référer au Huffington Post version américaine. La traduction des articles publiés, est toujours mentionnée avec une note au lecteur « Ce blog initialement publié sur le Huffington Post États-Unis a été traduit de l'anglais. »

Exemple:

5 http://rue89.nouvelobs.com/economie

6http://www.huffpostmaghreb.com/2015/04/10/game-of-thrones-saison-5-daeneryscollegue-ideale-tyrion-lannister-jon-snow-jaime-lannister-selon-sondage-monster_n_7036980. html 
Titre: 10 endroits parmi les plus colorés de la planète

Extrait: BEAUTÉ - Ces endroits existent. Pas dans un rêve ou une hallucination, ni même un film de Wes Anderson, mais ici, sur cette belle et mystérieuse planète... ${ }^{7}$

\section{Citations des sources originales}

Sur la page du Huffington Post Maghreb, nous notons que les liens, les sources et les documents sont en bleu ce qui nous permet en cliquant dessus de nous ramener à la source initiale comme par exemple le cas de l'article de sur « Game of Thrones » qui est en lien avec amnistie Internationale, et par un simple click, le lien dirige l'internaute vers le site officiel d'amnistie International www.amnesty.org.

Titre : «Game of Thrones» contre la vie réelle: 5 domaines où la réalité dépasse la fiction

"Malgré plusieurs exécutions, Westeros ne fait pas le poids comparé aux2 466 condamnations à mort recensées par Amnesty International dans le monde en 2014, chiffre marquant une augmentation de $28 \%$ par rapport à l'année précédente. ${ }^{8}$ "

\section{Structuration de l'information}

Les publications du Huffington Post sont construites avec une structuration solide, repérable, simple, facile et claire. Le titre, les auteurs, les dates de publications, les mises à jour, les icônes des réseaux sociaux invitent l'internaute à une lecture quotidienne du site.

ÉCOLOGIE - Le réchauffement planétaire menace la planète, les scientifiques tirent la sonnette d'alarme. Le point avec Mme Maria Sarraf, spécialiste de l'environnement, sur la situation.

La température mondiale risque d'augmenter de 4 degrés d'ici la fin du siècle. Par le passé, les scientifiques s'accordent à dire que la planète a connu des différentiels de températures semblables. Pensez-vous que l'on doive imputer cela à l'homme seul ou y a-t-il d'autres raisons? ${ }^{?}$

7http://www.huffpostmaghreb.com/purewow/10-endroits-parmi-les-plus-colores-de-laplanete_b_6855614.html

8 http://www.huffpostmaghreb.com/anna-neistat/game-of-thrones-contre-la_b_7049168.html 9http://www.huffpostmaghreb.com/2015/04/10/environnement-banque-mond_n_7038860. html 


\section{E. Affichage lisible des catégories d'informations disponibles: base de données, documents...}

Dans la mise en page du Huffington Post, les articles sont organisés, repérables et accessibles. La maquette du site guide l'internaute vers les informations les plus importantes de l'article, reprenant l'exemple de l'article « «Game of Thrones» contre la vie réelle: 5 domaines où la réalité dépasse la fiction ». Larticle est bien agencé avec des sous titres en gras et encadrés. Les chiffres et les faits sont eux aussi marqués en gras et bleu pour attirer l'attention.

\section{F. Liens proposés par le site: Choix et évaluation des liens : sont-ils pertinents, cohérent avec le contenu du site?}

Les liens sur le Huffington Post fonctionnent tels des documents de références. Revenir à la référence d'amnistie International, ou les liens proposés sont des documents, des statistiques et des publications d'amnistie internationale.

\section{IV.3. Axe 3: Ergonomie}

L'ergonomie est la façon de rendre un media participatif utilisable par le plus grand nombre de personnes avec un maximum de confort et d'efficacité. Evaluer l'ergonomie d'un site consiste à vérifier des normes de base en terme de facilite de navigation et de recherche pertinente d'information.

\section{A. Facilité de la navigation / orientation}

\section{Possibilité de télécharger des documents}

Une des principales composantes de l'ergonomie est l'accessibilité : le site doit être facilement et rapidement «pris en main»par un maximum de personnes (et pas réservé à une élite d'internautes «super-utilisateurs») et depuis n’importe quel ordinateur connecté.

\section{Url d'accès simple et intuitive}


Le site du Huffington Post Maghreb est facilement accessible, son url est simple, intuitive et donc facilement mémorisable. En effet, l'accès au site se fait soit en tapant l'url directement dans le navigateur, si l'internaute ne trouvent pas ils tentent alors une recherche dans un outil de recherche.

\section{Rapidité du temps de chargement des pages}

Internet est le média de l'instantané, par conséquent le temps de chargement des pages joue un rôle très important dans l'appréciation d'un site d'autant plus que la communication est payante pour la majorité des internautes. Le site du HP Maghreb cherche la visibilité du grand public et a pour objectif de créer du trafic et de toucher un maximum d'internautes, il est adapté à une connexion classique par modem $56 \mathrm{~K}$. Le temps de chargement d'une page d'accueil n'excède pas les cinq à dix secondes. Le HP permet à ses lecteurs de partager le contenu de son site sur les réseaux sociaux comme Facebook, Twitter, Google plus. Par contre l'internaute ne peut pas télécharger les articles ni les imprimer. Si un article l'intéresse, il peut le copier sur le logiciel Word et l'imprimer. En ce qui concerne le téléchargement de la page, Huffington Post offre une mise en page simple et légère ce qui facilite le téléchargement et rend les recherches plus rapides. Les logiciels tels que «Flash» «Animation» qui bloquent la fluidité ne sont pas présents. Compteurs, animations flash et autres gadgets ne figurent pas sur le site. Ils fatiguent les yeux et distraient les visiteurs de votre véritable contenu.

\section{Universalité des technologies utilisées}

L’emploi de technologies spécifiques de programmation, d'animation, de diffusion... nécessite une certaine prudence. En effet, bien que le plug-in Flash soit désormais présent sur la plupart des ordinateurs connectés, de nombreuses technologies ne sont pas encore devenues des standards. De même certaines ne fonctionne que sous un environnement spécifique. Il existe ainsi de nombreuses incompatibilité entre Internet Explorer et Netscape ou entre Windows et Mac OS... L'interface du site du HP Maghreb est accessible par tous et 
depuis n’importe qu’elle machine connectée.

\section{Respect des usages d'Internet}

Lors de la conception d'une interface web, il est important de se conformer aux usages d'Internet. En effet, comme dans toute interface, les utilisateurs sont plus ou moins conditionnés par leurs expériences passées et leur environnement. Le lien html du site Huffington Post Maghreb est mis dans un corps de texte et symbolisé par le soulignement du mot. De même, les éléments de navigation sont mis en haut et sur la gauche de la page, et le logo de l'entreprise en haut à gauche. Les internautes ont tellement l'habitude de se repérer dans ce type d'interface qu'il est devenu un usage (tout comme le fait de mettre la date en haut à droite d'une lettre). De plus, cette disposition suit le sens naturel et intuitif du cheminement visuel sur un écran : les yeux explorent l'écran en effectuant un «Z» s'arrêtant dans la zone centrale de l'écran.

\section{B. Design:}

1. Cohérence avec l'image de l'entreprise et de sa stratégie de communication on line

Le design du site Huffington Post Maghreb sert les objectifs marketings et la stratégie de communication globale de l'entreprise. Dans ce sens, la charte graphique du site reflète et communique une image et une identité visuelle notamment avec la couleur verte adaptée à sa stratégie. Ainsi, l'internaute retrouve sur toutes les versions du Huffington le même logo, les mêmes couleurs et la disposition similaires des articles.

\section{Charte graphique homogène}

L'utilisation du Huffington Post Maghreb est facile et l'apprentissage est rapide de la navigation au sein du site. La charte graphique est homogène sur toutes les pages du site. Dès la page d'accueil, l'internaute identifie les éléments de navigation et les zones de contenus, ce qui fidélise l'internaute et le rend loyal au site. 


\section{Eléments graphiques utiles}

Comme pour tout support de communication, les éléments graphiques doivent être au service de l'information. Dans le cas du site du Huffington Post Maghreb, l'utilisation de pictos, images, animations Flash... sert à l'illustration des propos et à l'habillage de l'interface. Les pages ne sont pas trop surchargées en éléments graphiques. Ainsi, le lecteur est guidé directement vers les zones et informations stratégiques.

\section{Navigation}

\section{Page d'accueil efficace}

Véritable vitrine du site Internet, la page d'accueil du HP Maghreb est traitée avec une attention toute particulière. Le logo est facilement repérable avec un accès rapide aux articles et aux rubriques. Les informations sont récentes et régulièrement mises à jour. Les zones de navigation sont lisibles et identifiables instantanément avec un lien qui mène directement aux mentions légales, au contact et à la rubrique " qui sommes-nous? Pour être efficace, un site doit proposer une navigation intuitive et cohérente. Ainsi, les internautes doivent faire un minimum d'efforts pour se repérer dans le site et trouver les informations qu'ils recherchent. Les éléments de navigation du site HP Maghreb respectent les logiques du parcours visuel. Dès l'affichage de la page d'accueil, les yeux font effectuer des mouvements de saccades qui vont former d'une manière générale un « $Z$ » partant du haut à gauche et se terminant vers le bas droit de l'écran. Le mouvement des yeux va naturellement être fonction de l'attractivité visuelle des éléments de la page. D’une manière générale, la zone centrale du HP Maghreb est le point de passage et de repos du regard et soigneusement respecter. A l'intérieur du site, les éléments de navigation facilitent la navigation descendante et transversale : descendre dans l'arborescence du site et passage aisé d'une rubrique à l'autre. Ainsi, l'accès à l'information recherchée au HP Maghreb est réduit à un minimum de temps. Les barres de navigation sont lisibles, les intitulés à la cible sont compréhensibles et adaptables. La fluidité de la navigation et la hiérarchisation des rubriques sont respectés. 
2. Recherche et accès facile et rapide à l'information

Plus qu'une simple vitrine, la page d'accueil doit présenter l'ensemble des principales rubriques du site. D'un seul coup d'œil, l'internaute doit pouvoir se faire une idée des contenus et fonctionnalités du site, et si possible y accéder en 1 clic.

Linterface web du Huffington Post Maghreb propose plusieurs accès et entrées dans les rubriques avec une identification facile et permanente des éléments de navigation et des liens html. Ainsi, le site s'adapte aux différentes typologies d'internautes et de recherche d'information.

Le Huffington Post offre un moteur de recherche interne guidant l'internaute dans toutes ses demandes, il se situe exactement au-dessous de son logo. L'internaute peut utiliser des mots clés dépendamment de son intérêt, et le moteur le guide vers toutes les publications en lien avec sa demande.

Il existe un autre champ pour la recherche en bas de chaque pages:

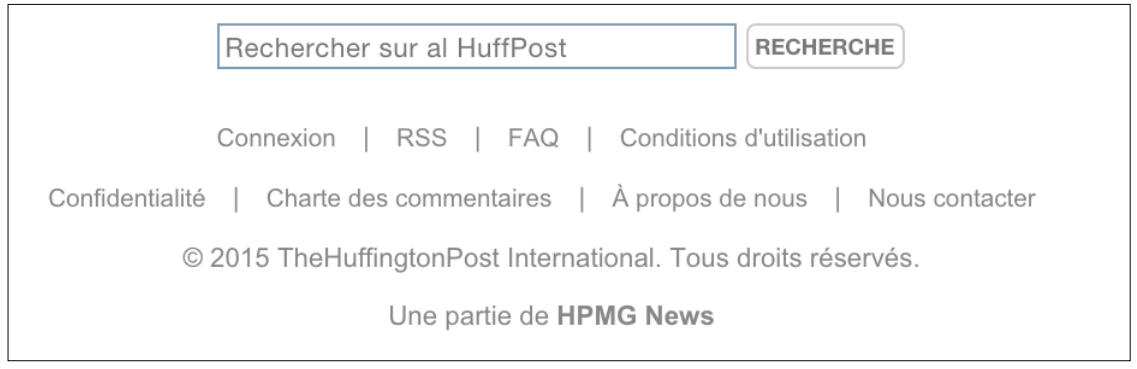

Figure 4 : Recherche en bas de page

3. Navigation avec un index ou un plan de classement

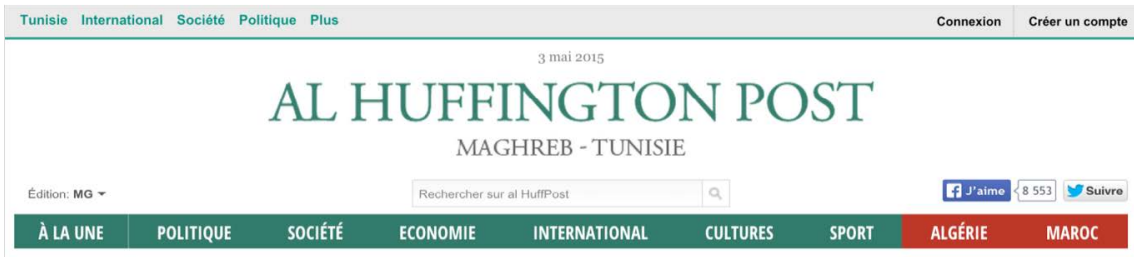

Figure 5: Navigation avec index de placement 
L'index de navigation et le plan de classement, se présentent clairement au début de la page d'accueil. L'index mène les lecteurs vers les publications en rapport avec le Maroc et l'Algérie et vice versa pour la page d'accueil du Huffington Tunisie.

4. Possibilité de retourner à la page d'accueil, présence d'un ascenseur

A n'importe quel moment le lecteur peut cliquer sur le logo du Huffington Post pour retourner à la page d'accueil.

Repérage facile dans l'arborescence

A tout moment et à n'importe quel endroit du site, l'internaute doit pouvoir visualiser rapidement:

- où il est?

- où il a déjà été?

- où il peut aller?

La page d'accueil est l'élément central et fondamental du site, elle doit ainsi orienter et guider l'internaute vers l'ensemble des contenus du site.

Dans l'interface du HP Maghreb le repérage n'est pas facile à l'intérieur du site, les barres de navigation ne sont pas visibles en permanence et noriente pas l'internaute vers les principales rubriques du site. D’autres éléments d'aide à la navigation y figurent:

- le plan du site, permet de visualiser l'ensemble des rubriques et l'arborescence du site.

- le «train d'arborescence» permet à l'internaute de visualiser à tout moment sa position dans l'arborescence.

- les boutons «retour», "précédent», «sommaire»... sont pas clairs. Même s'ils sont redondants avec les boutons du navigateur ou le «train d'arborescence», ils sont très utilisés par certains internautes alors que d'autres préfèrent utiliser le bouton «back» du navigateur. 


\section{Contenus rédactionnels}

Le surf sur le web est caractérisé par une navigation exploratoire rapide. Les internautes lisent les contenus rédactionnels des pages web en diagonale.

\section{Présence d’une ligne éditoriale}

Le style rédactionnel des contenus du Huffington Post Maghreb est adapté au positionnement du journal participatif généraliste. La ligne éditoriale correspond à l'atmosphère, au style général employé pour la rédaction de l'ensemble des contenus du site.

\section{Présence de plusieurs niveaux de lecture}

Le Huffington Post Maghreb propose différents niveaux de lecture afin de satisfaire les différentes typologies d'internautes : le lecteur pressé peut directement repérer les titres et les brefs résumés en page d'accueil, tandis que le lecteur intéressé peut accéder à l'intégralité de l'article par un lien «en savoir plus» ou «lire la suite». De même au sein d'une page, le texte est mis en forme et adapté au mode de lecture «en diagonale». Les techniques rédactionnelles adoptées par le Huffington Post Maghreb sont principalement:

- paragraphes et phrases courtes

- titres brefs et explicites

- hiérarchisation et illustration des informations (puces, tableaux, schémas...)

- liens html dans le texte dans la page (ancres) ou vers d'autres pages

- utilisation du Alt Texte : affichage d'un texte au passage de la souris sur un lien html

\section{Exhaustivité des contenus}

Internet est le média de l'information, gigantesque source de données et de connaissance. Lorsque l'internaute effectue une recherche sur le Huffington Post Maghreb il trouve une réponse à sa 
question. La mise en place d'informations est plutôt complète, exhaustive et régulièrement mise à jour.

\section{E. Fonctionnalités}

\section{Fonctionnalités «fonctionnelles»}

L'ensemble des fonctionnalités proposées sur le site doivent être conçues dans la même optique de simplicité et de confort d'utilisation. La mise en place d'un forum de discussion, d'un formulaire de contact, d'un moteur de recherche... figure sur le site du Huffington Post Maghreb.

\section{Fonctionnalités utiles et apportant une réelle valeur ajoutée}

Au-delà de l'utilisation de la fonctionnalité, l'»usabilité» comprend également la notion fondamentale de valeur ajoutée. En effet, même si l'accessibilité, le design, la navigation et les contenus sont parfaits, un site qui napporte pas une réelle valeur ajoutée à ses internautes ne fera pas la différence. La valeur ajoutée du Huffington Post Maghreb provient de l'ensemble des contenus, services et fonctionnalités qui répondent au besoin de l'internaute. Avec l'expérience positive de leur visite sur le site du HuffPost Maghreb, ils seront satisfaits et seront susceptibles de revenir ultérieurement sur le site, de s'y enregistrer, de le conseiller à un ami, de l'ajouter dans ses favoris.

\section{Conclusion}

Site de référence en ce qui concerne le journalisme participatif, le HP dans sa version maghrébine laisse la démarche de démocratisation du journalisme à la marge d'une architecture sophistiquée. En appliquant une méthodologie sémiotique et situationnelle, cet article essaie de montrer la conformité du langage et de l'esthétique du journalisme participatif mais montre que le site de Al HP échoue à afficher une ligne directrice claire pour le media.

En relayant une grande partie de ces articles d'autres medias ayant une assise professionnelle et une reconnaissance historique, Al HP se conforme à des normes scientifiques de présentation : le financement est indépendant, l'information est mise à jour régulièrement, l'accessibilité au contenu du site est facilitée par une arborescence claire 
et simple. Au niveau du contenu, le référencement de l'information est exhaustif, la langue est de qualité impeccable et l'information est correctement structurée. Enfin, l'ergonomie du site se base sur une fonctionnalité dans la navigation et la recherche d'information.

Cependant, il est impossible dans le cadre de cette recherche de dégager un marquage politique ou social pour Al HP. Alors que les versions anglo-saxones se démarquent par un franc-parler et des contributions de personnalités connues, la version maghrébine n’a pas de couleur spécifique. Le site peine à montrer une finalité politique ou sociale. Cette expérience pourtant unique dans le monde arabe ne se distingue pas ni par sa valeur ajoutée ni par la création d'une dynamique d'expression démocratique. La forte contribution de journalistes professionnels à la production d'articles rend problématique la contribution des journalistes-amateurs à cette plateforme. De plus, le financement par recettes publicitaires remet en question l'indépendance financière et donc politique de ce media.

Néanmoins, le rôle que peut jouer Al HP dans lélargissement des pratiques démocratiques est indéniable. Cette expérience devrait être généralisée, soutenue mais affranchie du diktat du financement par les groupes industriels et de l'opacité de l'accès à l'information.

\section{Références bibliographiques}

ALTERMAN, Eric. What Liberal Media. New York: Basic Books, 2003.

BAKER, C. Edwin. The Media that Citizens Need. University of Pennsylvania Law Review, 147(2), 1998. p. 317-409.

BELL, David. An Introduction to Cyberculture. London/New York: Routledge, 2003.

BOWMAN, Shayne ; WILLIS, Chris. We the Media : How Audiences are Shaping the Future of News and Information. The Media Center. 2003.

CHAMPLIN, Dell. ; KNOEDLER, Janet. The Media, the News, and Democracy : Revisiting the Dewey-Lippman Debate, Journal of Economic Issues, 40(1), 2006. p. 135-152. 
COVER, Rob. Audience inter/active. Interactive Media, Narrative Control And Reconceiving Audience History, New Media and Society, 8(1). 2006. p. 139-158.

DE ROSNAY, Joel. La révolte du pronétariat. Des Mass Média aux Média des masses. Paris : Fayard. 2006.

HEÏD, Marie-Caroline ; MELIANI, Valérie. La sémiotique situationnelle appliquée à l'évaluation qualitative de sites web, Actes du 2ème colloque international francophone sur les méthodes qualitatives 25 et 26 juin 2009 à Lille. 2009.

HERMIDA, Alfred ; DOMINGO, David; HEINONEN, Ari; PAULUSSEN, Steve; QUANDT, Thorsten.; REICH, Zvi; SINGER, Jane; VUJNOVIC, Marina. The Active Recipient: Participatory Journalism Through the Lens of the Dewey-Lippmann Debate. Paper presented to International Symposium on Online Journalism, University of Texas, Austin. 2001.

KIM, Eun-Gyo; HAMILTON, Jeffrey. Capitulation to Capital ? OhMyNews as alternative Media. Media, Culture and Society, 28(4). 2006. p. 541-560.

MORIN, Edgar. Introduction à la pensée complexe. Paris: ESF. 1990.

MUCCHIELLI, Alex. Approche par la contextualisation. Paris: A. Colin. 2005.

MUCCHIELLI, Alex. Manuel de sémiotique situationnelle pour l'interprétation des conduites et des communications. Montpellier: Le Moine Copiste. 2008.

PELISSIER, Nicolas ; CHAUDY, Serge. Le journalisme participatif et citoyen sur Internet: un populisme dans l'air du temps? Quaderni. Communication, Technologies, Pouvoir, 70. 2009. p.89-102.

REBILLARD, Franck. Le journalisme participatif, de l'idéologie à la pratique. Argumentum, 6. 2007. p. 11-23. 
REBILLARD, Franck. Création, contribution, recommandation: les strates du journalisme participatif. Les Cahiers du journalisme, 2223. 2011. p. 28-41. 